Umgebung seinen literarisch geprägten Wahrnehmungsgewohnheiten und Denkkategorien entsprechend fiktionalisiert und auch tatsächlich mit der Wirklichkeit kollidiert. Sein Schicksal wird allerdings nicht monokausal aus seiner quijotischen Disposition erklärt, sondern auch aus den sozio-politischen Gegebenheiten des Milieus, dem er entstammt, und aus den widerstreitenden historischen Kräften, die im Raum zwischen der fortschrittlichen, zivilisierten hischottischen Kultur auf ihn einwirken, wobei er diese aufgrund seiner lektürebedingten Wirklichkeitsblindheit freilich nicht erkennt. Scott knüpft auch insofern an die Don Quijote-Imitationen des 18. Jahrhunderts an, als die Desillusionierung des Helden gegen Ende des Romans als ein Aufwachen aus literarisch bestimmten Träumen dargestellt wird, ein Aufwachen, das den Blick für die Wirklichkeit öffnet.

Auf Flauberts Madame Bovary weist die sich in dem Scottschen Helden zeigende Tendenz vor, auf der Grundlage intensiver Lektüreerlebnisse und mit Hilfe einer lebendigen Einbildungskraft eine eskapistische Phantasiewelt zu erzeugen. Von dem romantischen Träumer Waverley, wic er in den ersten Kapiteln des Romans gezeichnet wird, ist der Weg nicht weit zu Emma Bovary, für die literarische Klischees das Material einer träumerischen Illusionsproduktion bilden.

Waverley steht auch insofern in der Cervantes-Tradition, als sich in dem hier effolgenden Durchbruch zu einer neuen Form des Erzählens ein bei Cervantes am Anfang der europäischen Romangeschichte paradigmatisch zu beobachtender Vorgang wiederholt, nämlich der, daß eine innovative realistische Romanform sich in der Auseinandersetzung mit der realitätsfernen Gattung der Romanze herausbildet. Die Affirmation des Wirklichen erfolgt bei Scott - ähnlich wie im Don Quijote und seinen Nachfolgern - gerade in der Darstellung eines Helden, der das wirkliche Leben im Sinne der illusionären Ideale und Handlungsschemata der Romanze interpretiert. Für Scotts spätere Schottlandromane ist das Modell des Don Quijote nicht mehr von Bedeutung. Nachdem ihm der Durchbruch zum historischen Roman gelungen war, war eine quijotische Heldenfigur nicht mehr erforderlich. Unter diesem Gesichtspunkt ist Waverley noch nicht der Ptototyp des historischen Romans. Es ist vielmehr ein Werk des Übergangs, in dem Scott eine frühere Phase seines Schaffens, in der er romantische Verserzählungen schuf, überwindet und sich von der Romantik löst und dem Realismus zuwendet. Daß er sich in dieser Situation eines quijotischen Helden bedient, ist ein weiterer Beweis dafür, wie tiefgreifend das Paradigma des Don Quijote die europäische Romangeschichte beeinflußt hat.

\section{MANFRED SCHMELING}

\section{Prometheus in Paris}

Komparatistische Überlegungen zum Ertrag der Intertextualitätsdebatte am Beispiel André Gides

Es gehört zu den Grundwahrheiten moderner Kulturwissenschaft, daß „Künstler und ihre Werke durch ihre bloße Zugehörigkeit zum intellektuellen Kräftefeld in Beziehung zu anderen Künstlern und deren Werken stehen" Pierre Bourdieu, den man als Vertreter der soziologischen Richtungen zitieren darf, glaubt, daß der Schaffende dieser Abhängigkeit mehr oder weniger passiv gegenübersteht ${ }^{2}$ : „weil er sich nämlich nicht bewußt ist, daß die Bildung, die er besitzt, ihn besitzt".

Das hier angedeutete allgemeine Problem des kulturellen Einflusses, dem sich, bei allem Bemühen um das ,Eigene', anscheinend kein individueller Schaffensprozeß entziehen kann, wurde längst literarisch diskutiert, bevor es wissenschaftlich in Mode kam. Und ebenso wie die Forschung, die in dieser Frage weder sachlich noch methodisch auf einen gemeinsamen Nenner zu bringen ist, zeigt sich auch die Literatur durchaus variabel in der Gewichtung des Themas. Stiller beispielsweise, Titelfigur des Romans von Max Frisch, lastet seine Identitätskrise als Künstler und Erzähler ausdrücklich den Gegebenheiten der modernen Gesellschaft $a^{3}$ : Wir leben in einem Zeitalter der Reproduktion. Einen C. G. Jung, einen Kafka oder einen Thomas Mann hat man in sich schon durch seine Bekannten, die ibrerseits auch bereits in lauter Plagiaten erleben. Ein halbes Jahrhundert vorher äußert André Gide in seinem Roman L'Immoraliste vergleichbare Gedanken - diesmal allerdings nicht aus der Perspektive eines von der Wiederholungsangst geplagten Melancholikers, sondern indem er Nietzsches Abrechnung mit zweitausendjähriger Kultur gleich sam in kleinem Maßstab verwirklicht: als die Tat eine Individuums (Michel), das den Ausbruch aus der, sekundären', von humanistischen 'Traditionen und Einflüssen geprägten Existenz ganz bewußt wagt ${ }^{4}$ : Je méprisai dès lors cet êtr secondaire, appris, que l'instruction avait dessiné par dessus. Il fallait sécouer ces surcharges. Et je me comparais aux palimpsestes; je goûtais la joie du savant, qui sous les écritures plus récentes, découvre, sur un même papier, un texte très ancien infiniment plus précieux.

1 Pierre Bourdieu: Zur Soziologie symbolischer Formen, Frankfurt/M. 1970, 115

2 Ebd. 120.

3 Max Frisch: Stiller. Roman (1953/54), Frankfurt/M. (Suhrkamp TB) 1976, 186.

L'Tmmoraliste, in: André Gide: Romans, Récits et Soties, CEuvres lyriques, Paris (Bibl. de la 
Der Vergleich mit dem ,Palimpsest ${ }^{`}$ besitzt heute seine besondere literaturwissenschaftliche Qualität als metaphorische Umschreibung intertextueller Zusammenhänge ${ }^{5}$. Und wenn Gide ihn zunächst auch in einem grundsätzlichen (existentiellen) Sinn benutzt, so entnehmen wir ihm doch zugleich die spezifische Betroffenheit des Dichters, der inmitten eines übermächtigen kulturellen Universums nach künstlerischer Selbstverwirklichung strebt. Dafür sprechen nicht nur die autobiographischen Bezüge des Immoraliste, sondern André Gide hat den ,Palimpsest'-Charakter der Kultur literarisch stets ganz gezielt in Szene gesetzt. Das „kulturell. Unbewußte“, wie Bourdieu es nennt, mag ein theoretisch reizvolles Gebiet insbesondere der tiefenhermeneutisch interessierten Literaturwissenschaft darstellen, die konkrete Beschäftigung mit Gide konfrontiert uns jedoch auch mit einem gegenteiligen Befund: Sein Erzähltex Le Prométhée mal enchaîné $(1899)^{6}$, schon der Titel deutet es an, ist geradezu ein Musterbeispiel für die textkonstituierende und sinnstiftende Funktion des kulturellen Erinnerungsvermögens, also des kulturell Bewußten.

Bevor wir uns dieser besonders interessanten Prometheus-Bearbeitung exemplarisch zu nähern versuchen, bedarf es einiger Vorüberlegungen zur Reichweite von Intertextualitätskonzeptionen. Es scheint nämlich an der Zeit, daß der theoretische Ertrag der Auseinandersetzung, mit seinen sachlichen und methodologischen Problemen, einmal explizit und systematisch in einen spezifisch komparatistischen Fragezusammenhang einbezogen wird ${ }^{7}$. Die von Komparatisten immer wieder postulierten Berührungspunkte zwischen Allgemeiner und Vergleichender Literaturwissenschaft ergeben sich ja vor allem dort, wo empirische Einzelbeobachtungen über literarische Verflechtungen in theoretische Aussagen münden. Und hier dürfte sich das angesprochene Forschungsgebiet, insbesondere was das Textverständnis, die typologischen und entwicklungsgeschichtlichen Aspekte betrifft, als besonders fruchtbar erweisen ${ }^{8}$.

Seit die modernen Literatur- und Textwissenschaften begonnen haben, die Beziehungen zwischen Text und Prae-Text genauer auszuloten, sind über zwei Jahrzehnte vergangen. Zwar scheint die Diskussion nach einer stärke theoretisch orientierten Phase nun in eine Phase der systematischen Überprüfung und konkreten Anwendung eingetreten zu sein. Aber im Prinzip ist die ,Lust am Intertext ${ }^{t 9}$ ungetrübt. Das gilt freilich erst recht für die literarische

${ }^{5}$ Vgl. Gérard Genette: Palimpsestes - La litt. au second degré, Paris 1982.

6 Wir zitieren diesen 'Text nach folgender Ausgabe: Le Prométhée mal enchaîné, Paris (Gallimard) 1980 .

Es geht also nicht darum, die Diskussion noch einmal grundsätzlich aufzurollen. Einen besonders klaren wissenschaftsgeschichtlichen Überblick liefert inzwischen Manfred Pfister: Konzepte der Intertextualität, in: Intertextualität - Formen, Funktionen, anglistisch Fallstudien, hg. v. U. Broich und M. Pfister, München 1985, 1-30.

8 Zum komparatistischen Stellenwert der Intertextualitätsdiskussion vgl. auch Vf.: Textuell Fremdbestimmung und lit. Vergleich, in: Neobelicon 12, H. 1 (1985), $231-239$ (= FS Jacque Voisine)

9 Vgl. Le plaisir de lintertexte, hg. v. R. Theis und H.T. Siepe (Referate des Duisburger Colloquiums), Frankfurt/M./Bern/New York 1986. Det Titel des Sammelbandes demonstriert in actu, wovon er spricht, indem er an Roland Barthes (Le plaisir du texte,
Praxis selber, die von jeher neben vielen anderen Konkretisationen auch intertextuelle Lesarten zuläßt. Denn die Intertextualität präsentiert sich uns nicht als eine historische oder individuell begrenzte textuelle Sonderform, sondern ist grundsätzlich immer vorhanden (wobei es natürlich zu bestimmten epochenspezifischen bzw. typologischen Entwicklungen kommen kann). Der Rezipient muß sie gewissermaßen ,abrufen', und er tut das - aus Gründen, über die, wenigstens andeutungsweise, noch zu sprechen sein wird - in diesen Jahrzehnten besonders häufig.

Der Begriff ,Lesart' darf indes nicht mißverstanden werden. Auch wenn er nahelegt, daß hier ein bestimmtes Erkenntnisinteresse des Rezipienten vorliegt und weniger eine spezifische vom Text bzw. vom Autor ausgehende Intention, so ist das kein Plädoyer für freies Assoziieren: Fast jeder Text enthält in der einen oder anderen Form Signale, die den Vorgang der Rezeption intertextueller Prozesse lenken, sei es auf der Ebene von Einzeltextreferenzen mit konkreten Markierungen der,Quelle' (Peter Weiss: Der neue Prozeß u. a.), sei es in der allgemeineren Form der Systemreferenz, z. B. der Markierung von Gattungszugehörigkeit bzw. -wechsel, von literarischen Stereotypen, etwa Mythen, Motiven u. v.m. ${ }^{10}$. Man kann vorwegnehmen, daß unser Gide-Beispiel primär systemorientiert ist; der Prometheus-Mythos wird in diesem Werk als sensus communis ebenso vorausgesetzt wie bestimmte Gattungstraditionen oder Textsorten, mit denen der Autor sein ironisches Spiel treibt

Ein solcher, weitgehend empirisch überprüfbarer Intertextualitätsbegriff ist maximalistischen Konzeptionen sicherlich vorzuziehen. Poststrukturale oder dekonstruktionistische Auffassungen, wonach jedem Text aufgrund der Teilhabe an einem universellen Intertext die Negation seiner Identität gleichsam in die Wiege gelegt sei, haben der theoretischen Diskussion über literarische Seins- und Funktionsweisen zwar durchaus neue Impulse gegeben, aber sie lassen sich nur bedingt für die Analyse konkreter Bezüge nutzbar machen. Vor allem wird durch solche Verabsolutierungen leicht der Blick auf die besonderen literaturhistorischen bzw. -kritischen Entwicklungen verstellt Denn mit der textuellen Doppelbödigkeit literarischer Werke sind ja oft evolutionistische Vorgänge verbunden, die es herauszuarbeiten gilt. Wenn der Held in Cervantes' Don Ouijote zum fiktiven Gefecht gegen Windmühlen antritt, so verbirgt sich dahinter die sehr reale Auseinandersetzung mit der Tradition des Ritterromans und seinen Idealen. Literarisches Medium ist hiet unter anderem die Parodie, jene „Kampfarena zweier Stimmen“ (Bachtin), für die sich die Intertextualitätsforschung aus verständlichen Gründen ganz besonders interessiert. Und wenn der Autor von Madame Bovary mit dem ehebrecherischen Verhalten seiner Titelgestalt den Spätzeitcharakter romantischer Lektüre ins Bewußtsein hebt, dann ist das ein weiteres berühmtes Beispiel dafür, wie gleichsam an den Schnittpunkten von Text-Text-Bezügen

Paris 1973) erinnert. Übrigens schildert bereits Gide - in dem erwähnten Zitat au L'Immoraliste - jenen ,Genuß', den die Dechiffrierung des ,Palimpsests' bereitet.

${ }^{10}$ Zur Typologie vgl. ausführlich Ulrich Broich im Sammelband Intertextualität, aaO [Anm. 7] $31 \mathrm{ff}$. 
literaturspezifische - aber in gesellschaftliche Prozesse eingebundene - Konflikte und Veränderungen vergegenwärtigt werden.

Die Diskussionen über die Reichweite und die Formen intertextueller Beziehungen haben aus komparatistischer Sicht freilich ihren besonderen Reiz. Denn einerseits bestätigen sie auf ihre Weise viele für die internationale Literaturbetrachtung schon immer selbstverständliche Tatsachen und Praktiken, andererseits setzen sie, insbesondere was Methodologie und Systematik betrifft, auch neue Akzente. Sachliche Berührungspunkte ergeben sich bereits auf sehr allgemeinem Niveau. Dazu vier Punkte: Was beispielsweise die Forderung nach Dynamisierung des Literaturbegriffs betrifft, so ist die Entgrenzung des Textes in Richtung auf andere Texte ja geradezu die logische Konsequenz jeder vergleichenden Perspektive. Als literaturwissenschaftliche Vorgehensweise funktioniert der Vergleich immer dort, wo ein gegebener Text aufgrund entsprechender Markierungen einem konkreten Vorläufermodell, identifizierbaren anderen Texten, einem bestimmten literarischen, z. B. gattungsspezifischen System, einem anderen künstlerischen Medium etc. gegenübergestellt werden kann. Sofern - wie teilweise in der psychoanalytisch ausgerichteten Intertextualitätsforschung (Kristeva) - nur noch der Dezentralisierungs- oder Entgrenzungsprozeß als solcher, der Text als Prinzip der Produktion unendlicher, sich ständig selbst negierender Bedeutung zur Diskussion steht, ist dem Vergleich seine sachliche Grundlage allerdings weitgehend entzogen.

Zweitens: Die Tatsache, daß intertextuelle Beziehungen häufig auf fremde Literaturen und Kulturen übergreifen, tangiert wieder unmittelbar komparatistische Interessen. Während jedoch die Internationalisierung keine conditio sine qua non für die Entgrenzung des Textes darstellt (Beispiel: Plenzdorfs Werther), bedingt umgekehrt der internationale Rahmen einer Beziehung (Beispiel: Baudelaires Poe-Rezeption) notwendigerweise die Infragestellung textueller Identität. Letzteres bedeutet nicht, daß die innerhalb einer Nationalliteratur realisierten intertextuellen Prozesse grundsätzlich ein geringeres $\mathrm{Ma} \beta$ an Fremdbestimmung aufwiesen ${ }^{11}$ : „Die Fremdheitsmomente spielen bei Rezeption historischer Texte oft eine größere Rolle als bei der Rezeption ausländischer Texte". Die Auswirkungen von Vermittlungsprozessen, die speziell auf internationaler Ebene entstehen - Übersetzung, internationale Kritik u. ä. - , darf man aber nicht unterschätzen. Es hat sicherlich nichts mit „Ideologie“"11a zu tun, wenn man z. B. feststellt, daß Kafkas Wirkung auf den modernen französischen Roman (von Camus bis Robbe-Grillet) erst über den (tendenziösen) Transfer in die französische Sprache und eine in Frankreich gerade besonders aktive existentialistische Kritik möglich wurde. Und wo über die Literatur Beziehungen zu anderen Sprach- und Kulturräumen hergestellt wer-

11 Gunter Grimm: Rezeptionsgesch., München 1977, 134. Grimm spricht von der „Überbewertung der Sprache als eines Rezeptions- und Produktions-Räume (= Kulturräume) konstituierenden Elementes. Gerade hier könnten historische Rezeptionsuntersuchungen die Problematik dieser Ideologie aufzeigen".

${ }^{11 \mathrm{a}}$ Ebd. 154 den, kommt ja die historische Differenz zwischen frühem und spätem Werk als Entgrenzungsmerkmal meistens noch hinzu.

Drittens: Die Kompliziertheit solcher Text-Text-Bezüge setzt präzise analytische Kategorien voraus. Auf dem Gebiet der Sys tematik zeigen textwissenschaftlich orientierte Untersuchungen - wohl aufgrund des ausgeprägteren ,strukturalen' Bewußtseins - sicherlich mehr Engagement als manche hermeneutisch oder historisch ausgerichteten Forschungen. Das spiegelt sich zum Teil in den Taxinomien wider: Der Ausdruck, das Eigene und das Fremde verweist eher auf ein Problem der Hermeneutik, die Begriffe ,Intertext' oder ,Intertextualität' suggerieren ein System von Relationen (obschon die Termini in der wissenschaftlichen Praxis häufig ausgetauscht werden).

Erste, spezifisch komparatistische Ansätze zu einem solchen System liefert das allgemeine Modell von Dionýz Durišin, das die Bedingungen und die Formen „interliterarischer Beziehungen“ genauer zu erfassen sucht. Für die behutsam ideologische (marxistische) Einbettung des Modells sorgt ein bestimmter Weltliteratur-Begriff. Unter Weltliteratur versteht $\breve{D u r i s ̌ i n}^{12}$ „einen Komplex entwicklungsgeschichtlich und typologisch in gewisser Weise gegenseitig bedingter literarischer Schöpfungen". Interliterarische Beziehungen sind also kein Zufall und auch nicht einfach das Resultat individueller schöpferischer Interessen, sondern die gleichsam unvermeidliche Konsequenz weltliterarischer bzw. -historischer Gesetzmäßigkeiten. Ein solches Weltliteratur-Konzept kommt universalistischen Vorstellungen von Intertextualität durchaus nahe, auch wenn die ideologischen Voraussetzungen andere sein mögen. Das allgemeine Konzept Durišins mündet dann allerdings vor allem in systematisch-praktische Überlegungen über interliterarische Zusammenhänge - und hier vor allem besitzt sein Entwurf gewisse Meriten., Vergleichbar' sind Texte laut D̆urišin auf genetischer und/oder typologischer Grundlage. Genetisch bedingte Beziehungen entstehen teils durch externe Kontakte (z. B. durch das persönliche Gespräch, Reisen u. ä.), teils materialisieren sie sich intern ( $z$. B. in Übersetzungen, durch Zitat, Parodie u. ä.). Auf typologischer Ebene macht Durišin, unter Verwendung eines etwas fragwürdigen Typologie-Begriffs, gesellschaftlich-, psychologisch- und literarisch-typologische Zusammenhänge geltend. Mit anderen Worten: es geht ihm nicht nur darum, bestimmte konkrete Beziehungsformen (mit einer zumindest angedeuteten Hierarchisierung der Intensität intertextueller Kontakte) herauszuarbeiten, sondern damit verbunden ist beispielsweise die Frage, aufgrund welcher gesellschaftlichen Entwicklungen und Gesetzmäßigkeiten solche Kontakte möglich werden und wie sie zu beurteilen sind. Manche der komparatistischen Einzelkategorien, z. B. die Begriffe „Impuls“, „Kongruenz“, „Ähnlichkeit“, bleiben freilich vage; hier zeigt sich der Fortschritt moderner linguistisch orientierter Intertextualitätsforschung, die über den präziseren Beschreibungsapparat verfügt. Dabei scheinen

${ }^{12}$ Dionýz Durišin: Die wichtigsten Typen lit. Bežiebungen und Zusammenbänge, in: Aktuelle Probleme der vgl. Lit.forscbung, hg. v. G. Ziegengeist, Berlin 1968, 47-58, hier 47. Vg1. auch die ausfühtliche theoretische Diskussion komparatistischer Probleme, in: D. Dutišin: $\checkmark$ gl. Lit.forscbung, Berlin 1976. 
unterschiedliche Analysemethoden zunächst an gemeinsame sachliche Voraussetzungen anzuknüpfen: Man stellt u.a. fest, daß sich auch Durišin um jene traditionsreichen literarischen Formen bemüht, die seit Bachtins bahnbrechenden Untersuchungen über das „zweistimmige Wort" ${ }^{*} \mathrm{zu}$ den favorisierten Gegenständen der Dialog- bzw. Intertextualitätsforschung zählen: die Parodie, das Zitat, das Motto, die Anspielung u. a. m. - also Textsorten bzw. textuelle Äußerungen, die eine von anderen Texten unabhängige Existenz, eine eigene Identität, per definitionem nicht besitzen.

Auf der anderen Seite - und damit sei ein letzter theoretischer Aspekt angesprochen - muß man einfach zur Kenntnis nehmen, daß sich das Phänomen der Intertextualität nicht auf jene, teilweise schon aus der antiken Poetik bekannten klassischen Beziehungsformen reduzieren. läßt. Dem widersprechen aktuelle literarische Entwicklungen. Vielfach geht es den modernen Autoren ja gar nicht mehr um die Korrektur, die Adaption, die Parodie oder um sonstiges Aus-einander-Setzen mit fremder literarischer Äußerung in gleichsam geordneten rhetorischen Bahnen. Sondern das Phänomen hat sich verselbständigt zum intertextuellen Diskurs, der sein Ziel in sich selbst sucht. TextText-Bezüge gehören seit eh und je zur literarischen Praxis (übrigens ohne $\mathrm{da} \beta$ diese Praxis besondere wissenschaftliche Diskussionen hervorgerufen hätte), aber erst im Verlaufe des 20. Jahrhunderts und vor allem unter dem Einfluß der ,Tel Quel'-Bewegung und des, Nouveau (nouveau) Roman' entwikkelt sich daraus eine spezifische Schreibweise; und diese besondere Schreibweise, in der sich Theorie und Praxis gegenseitig durchdringen, dürfte entscheidend zum wissenschaftlichen Erfolg des Phänomens beigetragen haben. Intertextualität als durchkonstruiertes künstlerisches Verfahren und Intertextualität als mehr oder weniger bewußte Auseinandersetzung mit Einzeltexten, Gattungen, Themen, fremden Medien usw. schließen freilich einander nicht aus. Unser Beispiel, André Gides Le Prométbée mal enchaîné, vereinigt beide Tendenzen in einem einzigen Text.

Damit verlagert sich das komparatistische Erkenntnisinteresse zwangsläufig auf eine andere Ebene. Nicht die durch den gegebenen Text jeweils wieder aufgenommenen (und mit ihm gegebenenfalls zu vergleichenden) anderen Werke, auch nicht dieser oder jener texttypologische Bezug (,abstrakter' PraeText) treten ins Bewußtsein. Vielmehr wird unser kritisches Augenmerk auf die Tatsache gelenkt, daß hier offenbar neue Möglichkeiten künstlerischer Gestaltung, etwa der totalen Entgrenzung und Dezentralisierung des Textes, ausprobiert werden. Inwieweit letzteres wirklich als Modernitätskriterium gelten, ja - wie manche glauben - als ,postmodernes' Symptom einer sich ,erschöpfenden' Literatur betrachtet werden kann, ist ein durchaus ernstzunehmendes Problem, über das man gerade erst nachzudenken beginnt ${ }^{13}$.

3 Vgl. etwa Wolfgang Karrer: „Vor allem in der modernen und postmodernen Literatu sind intertextuelle Verfahren beliebt geworden, bei denen Strukturen oder Relationen aus Pratexten ubernommen werden". Intertextualitat als Elementen- und Struktur-Reproduktion, in: Intertextualität, aaO. [Anm. 7] 99. Vgl. dazu die weitergehende, auf der, Erschöpfungs' Theorie (,Literatur of Exhaustion“) von John Barth fußende These „Postmoderne und
Wenden wir uns nun konkret unserem Paradigma zu: André Gide und seiner Erzählung Le Prométbée mal enchaîné. Auf der Grundlage der vorgenommenen theoretischen Fixierungen soll versucht werden, ein komplexes Intertextualitätsprofil herauszuarbeiten: die genetischen Vorgänge (Gide als Rezipient fremder Literatur), die Formen der literarischen, d.h. produktiven Vermittlung solcher Vorgänge, die Rolle, die dabei die herrschenden (z.B. gesellschaftlichen oder literarischen) Entwicklungstendenzen spielen, und schließlich der intertextuelle Prozeß als spezielle - modernistische - Schreibweise.

Die intertextuelle Lesart bietet sich in diesem Fall nicht nur an, sondern scheint unumgänglich. Ohne den literarischen Hintergrund der prometheischen Tradition bleibt uns verschlossen, was den Text als kontrovers oder gar als subversiv auszeichnet. Letzteres wird unmittelbar durch den Titel und die Ausgangssituation der Erzählung verdeutlicht: Scblecbt gefesselt ist dieser Prometheus in der Tat, denn er hat seine Ketten längst abgestreift und den Kaukasus der Sage gegen das Paris der Jahrhundertwende eingetauscht: Der Titan als Flaneur, auf einem Boulevard der französischen Metropole, nach Menschen Ausschau haltend. Aktualisierende Transpositionen vom Fernen zum Nahen sind bei Mythenbearbeitungen nichts Außergewöhnliches, sondern fast die Norm. Dennoch dürfte sich kaum ein Mythos besser zur Thematisierung insbesondere zeitgenössischer Erscheinungen eignen als gerade der für ,Schöpferkraft', ,Revolte', ,Erkenntnis', ,Freiheit' oder ,Fortschritt' stehende prometheische Held.

Gides verschachtelter Prométhée läßt sich nicht linear wie eine Intrige nacherzählen. Der Schauplatz, der die verschiedenen historisch bzw. mythologisch disparaten Ereignisse mit der Gegenwart verknüpft, ist, wie gesagt, Paris, insbesondere ein zwischen der ,Madeleine' und der, Opera' gelegenes Café. In dieser Umgebung geschieht, wie der Erzähler es formuliert, Merkwürdiges. Zeus, der Bankier, auch le Miglionnaire genannt, verpaßt einem xbeliebigen Herrn, der ihm das verlorene Taschentuch aufhebt und auf einen mit 500 Franken gefüllten Umschlag eine $x$-beliebige Adresse schreibt, zum Dank eine schallende Ohrfeige. In der Folge der Sequenzen erlebt man dann Prometheus, vom Kaukasus kommend, nun arbeitsloser Streichholzfabrikant, im Dialog mit dem Kellner, der seinerseits für die Knüpfung zwischenmenschlicher Kontakte verantwortlich zeichnet. Coclès, der zufällige Empfänger der Ohrfeige, und Damoclès, der zufällige Empfänger der 500 Franken, tauchen auf, anschließend ein Adler, der Coclès ein Auge und Prometheus ein Stück Leber heraushackt. Bemerkenswert ist, daß Prometheus die Leber später freiwillig anbietet, Zeus also mit dieser Sache nichts mehr zu tun hat. Damoclès stirbt an der Bürde des unverdienten Geldes, Coclès macht ein Heim für

Intertextualität scheinen also tatsächlich so etwas wie Synonyme zu bilden" bei Joseph C. Schöpp: ,Endmesbed in endtanglements" - Intertextualität in Donald Bartbelmes ,The Dead Father', in: Intertextualität, aaO. [Anm. 7] 333. 
Einäugige auf. In einem rhetorischen Auftritt vor großem Publikum berichtet Prometheus über sein Verhältnis zum Adler, der, weil gut genährt, immer schöner wird. Das Ganze endet schließlich mit einem Diner, an dem Prometheus, Coclès und der Kellner teilnehmen. Auf dem Speiseplan steht - der Adler (116): s'il m'eût fait moins souffrir il ê̂t été moins gras; moins gras il ê̂t été moins déléctable.

Die beiden zentralen Motive dieser modernen Allegorie seien gleich genannt: Es sind der acte gratuit, d.h. die unmotivierte Handlung, die wie der Blitz in eine moralisch motivierte Welt einschlägt, und die Geschichte des Adlers als Geschichte der Menschheit. Freilich nicht irgendeiner Menschheit, sondern der immer schon in Schuld (500 Franken!) und Sünde verstrickten, der in die Pflicht genommenen unfreien Menschheit. Der Adler repräsentiert bei Gide also vor allem innere Zwänge, die Skrupel, das Gewissen, ja das Bewußtsein schlechthin (71): Notre aigle est notre raison d'être, Messieurs, sagt Prometheus.

Die Rolle von Zeus bleibt ausgesprochen ambivalent. Es bietet sich an, das Geld, das er aus purer Spielfreude, ohne jedes Interesse am Menschen, wie es heißt, in Umlauf bringt, als Symbol ökonomischer Macht und die davon Betroffenen als Opfer kapitalistischer Entfremdung zu deuten. Aber Gide ist genau das Gegenteil eines dogmatischen Denkers und verwickelt den hermeneutisch interessierten Leser in Widersprüche, die, wie wir am Ende sehen werden, letztlich unaufgelöst bleiben müssen. Zeus wird uns zugleich als le bon Dieu (87) präsentiert, der - wie der alttestamentliche Gott leiden läßt. Erst einmal in Gang gesetzt, bekommt seine Gratisaktion eine deterministische Qualität, der sich der Mensch nicht entziehen kann. Gide knüpft also durchaus an typische Konstituenten der Prometheus-Exegese an, wie sie uns aus der literarischen Tradition seit der Antike bekannt sind.

Die Frage, auf welchen äußeren und inneren Kontakten die intertextuellen Beziehungen in Gides Prométbée mal enchaîné beruhen, ist unter anderem deshalb nicht ganz leicht zu beantworten, weil der Autor schon früh über ein enormes kulturelles Wissen verfügte, das durch diesen Text eher implizit als explizit absorbiert und transformiert wurde. Trotzdem kann, was das PrometheusThema betrifft, die Anreger-Funktion insbesondere von Aischylos, Goethe und Shelley als gesichert gelten. Shelley z. B. ist nicht nur in Gides Tagebuch, sondern vor allem auch im Prometheus-Text selber gegenwärtig, und zwar über das Asia-Motiv, jene romantische, das Licht des Lebens repräsentierende Gestalt, die den Helden des Prometheus unbound gegen Jupiter, den Gott des Bösen, unterstützt.

Die andere wichtige rezeptionsgeschichtliche Station ist die Begegnung mit Goethe. Bereits 1892 hat Gide das Prometheus-Fragment und die Ode gelesen $^{14}$, und Jahre später spricht er von seinen Schwierigkeiten, sie zu übersetzen, und fügt hinzu ${ }^{15}$ : Il me semble qu'aucun coup de ciseau, pour dégager

${ }_{14}$ André Gide: Journal 1889-1939, Paris (Bibl. de la Pléiade) 30: Lu des poésies de Goethe; le Prométbée $[\ldots]$.
15 Vgl. ebd. 906. ma figure intérieur n'a enfoncé plus avant (même ceux de Nietz̧sche par la suite) que ne firent, lorsque je les lus pour la première fois à vingt ans, ces vers admirables du Prométhée. Es fällt auf, daß sich Gide auf betont dialektische Weise zur Einflußfrage äußert und die Bewunderung des Vorbilds immer mit dem stolzen Anspruch auf eine eigenständige, individuelle Entwicklung als Künstler ver$\mathrm{knüpft}^{16}$ : Mais sied-il de parler ici d'influence? Si je me laissais instruire par Goethe si volontiers, c'est qu'il m'informait de moi-même. So behutsam man bei der Einbezichung von Goethes Prometheus-Deutung vorgehen muß, so gewiß ist, daß auch diese Filiation in der Antwortstruktur des Gide-Textes ihre Rolle spielt ${ }^{17}$ : dans son Prométbée [...] j’apprenais déjà que rien de grand ne fut tenté par l'bomme, qu'en révolte contre les dieux. Die Frage ist natürlich, welche Art von Göttlichkeit Goethe und welche Gide meint. Wenn Goethe im nachhinein (1813, in Dichtung und Wabrbeit) von der Ode als vom Zündkraut einer Explosion spricht und in einem Karlsbader Brief an Zelter von der revolutionären Jugend, der sein Prometheus als Evangelium recbt willkommen sein möchte ${ }^{18}$, so beweist dies, daß die wirkungsgeschichtliche Potenz des Prometheus jedenfalls über den religiösen und poetischen Freiheits-Gedanken hinausweist.

Eine weitere, nur mittelbar das Prometheus-Problem betreffende Beziehung, bedarf im Zusammenhang mit der historisch-genetischen Beweisführung zumindest der Erwähnung, nämlich die Auseinandersetzung mit Dostoevskij. In Le Prométhée mal enchaîné hat sie insofern ihre Spuren hinterlassen, als die Idee der Gratisaktion bereits in den Werken des russischen Dichters vorgebildet ist, etwa in Scbuld und Sübne. Raskolnikovs Philosophie des Bösen - obne Kasuistik töten, nur für mich töten, für mich allein! ${ }^{19}$ - spielt als Prae-Text, nicht nur des Prometheus, sondern beispielsweise auch des großen Romans Les caves du vatican eine durchaus ernst zu nehmende Rolle. Auch in bezug auf sein Verhältnis zu Dostoevskij glaubt Gide sich im übrigen absichern zu müssen ${ }^{20}:$ Dostoievski ne m'est souvent ici qu'un prétexte pour exprimer mes propres pensées. Der französische Ausdruck prétexte (Anlaß oder Vorwand) bekommt hier praktisch eine wörtliche Bedeutung als ,Prae-Text'. Gides Äußerungen über Goethe und Dostoevskij geben uns somit bereits eine erste Vorstellung davon, wie er selber

16 André Gide: Goethe, in: Nouvelle Rev. Franc. 38 (1932), 373.

17 Ebd. 375.

${ }_{18}$ Vgl. Johann Wolfgang Goethe: Worke V, Berlin/Weimar 1964, 683.

${ }_{19}$ Fjodor M. Dostojewskij: Scbuld und Sübne, München (dtv Klassik) 1984. Vgl. 535.

20 Die entsprechende Äußerung findet man in den Conférences du Vieux-Colombier (1923), in: André Gide: Dostoievski - Articles et Causeries, Paris 1964, 206. Den Prozeß der literarischen Tranformation enter denen Montagne spricht, habe er den Womponenten entnommen: te qui converait a mon miel (ebd. 206). Dazu gehort insbesondere die Idee des acte gratuit, die Gide im selben Artikel genauer ausfuhrt (219). Was den Einfluß-Gedanken als interkulturelles Problem betriff, so ist auch folgende Bemerkung zur französischen Dostoevskij-Rezeption aufschlußreich. Gide beklagt nationale Vorurteile (229): $A$ ne vouloir admettre de l'étranger que ce qui déjà nous ressemble, où nous puissions trouver notre ordre, notre logique, et, en quelque sorte, notre image, nous commettons une grave erreur. Zur DostoevskijRezeption bei Gide vgl. den Artikel von István Fodor: Dostoievski et André Gide, in: Europe 49 (1971), no. 510, 106-112. 
sich den intertextuellen Dialog vorstellt: Nicht einfach als fremdkulturelle Überwältigung, als Einfluß von außen, sondern als einen vom individuellen Fragehorizont und Formwillen des rezipierenden Autors abhängigen Transformationsprozeß. Damit drückt Gide auf seine Weise, subtiler vielleicht als manche komparatistischen Theorien den Unterschied zwischen Einfluß und Rezeption aus. Was er uns in diesem Zusammenhang freilich unterschlägt, ist die Tatsache, daß ein solcher Fragehorizont zugleich auf allgemeinen literarischen Entwicklungstendenzen und gesellschaftlichen Erscheinungen beruht. Dazu gleich mehr.

Wie wirkt sich Gides Rendezvous mit der west- und osteuropäischen Kultur nun konkret auf die Produktionsstrategien seiner Prometheus-Bearbeitung aus? Er benutzt zunächst drei klassische, oft ineinander übergehende Verfahrensweisen, die uns die Überlagerung verschiedener Texte signalisieren, nämlich die Reminiszenz, das Zitat und die Parodie. Das kulturelle Reservoir, mit dem diese Verfahrensweisen korreliert sind, ist außerordentlich komplex. Dazu gehören neben der Tradition der Prometheus-Überlieferung vor allem das Alte und Neue Testament, die Vergilschen Eklogen, denen Gide bestimmte Protagonisten wie Tityre (Tityrus), Ménalque (Menalcas) und Moelibée (Meliboeus) entlehnt, sowie eine Reihe weiterer mythologischer Gestalten, die den verschiedensten Sagenkreisen entnommen sind. Damit nicht genug, baut er zusätzliche Textsorten ein und setzt so die intertextuelle Auseinandersetzung auf einer mehr theoretischen Ebene fort: nämlich die Chronik, das Interview und den Vortrag vor dem Publikum. Sic werden explizit eingeführt als Chronique de la moralité privée, als L'interview du Miglionnaire (Interview mit Zeus also) und in Form einer Plakat-Ankündigung: $C e$ soir à 8 beures dans la salle des Nouvelles Lunes Promethée délivré parlera de son aigle. Hinzu kommt noch das Gleichnis, das als die eingeschobene Histoire de Tityre die Prometheus-Handlung gleichsam metaphorisch nachinszeniert und daher also auch intratextuell wirksam ist.

Es ist reine Ironie, daß Gide hier auf Formen zurückgreift, die eher pragmatisch bestimmt sind, d. h. eine informative (Chronik, Interview), persuasive (Vortrag oder Ansprache) oder didaktische Funktion (Gleichnis) erfüllen ${ }^{21}$. Denn in ihrer Zweckgerichtetheit sind sie der Gideschen Ästhetik, die jeden affirmativen Diskurs und jeden Pragmatismus der Kunst ablehnt, genau entgegengesetzt. Vor diesem Hintergrund scheint es bedeutsam, daß der Autor seinen Text in der Ausgabe von 1914 erstmals als sotie, d.h. als eine Art Narrenspiel versteht und damit auf ein spätmittelalterliches Genre zurückgreift - wiederum eine Form intertextueller Verflechtung auf der Basis gattungsspezifischer Zeichensysteme - , in dem nicht nur ästhetisch-dramaturgisch ein erstaunliches $\mathrm{Maß}$ an Freiheit herrscht, sondern darüber hinaus äußerst despektierlich mit weltlichen und kirchlichen Herrschaftsformen umge-

${ }^{21}$ Diese funktionale Gliederung stützt sich - mit leichten Abweichungen - auf den Gliederungsvorschlag von Elaine D. Cancalon: Les formes du discours dans, Le Prométbé mal encbainét, in: Bull. des amis d" André Gide, Vol. IX, Nr. 49, Jan. 1981, 35-44, vgl. 39. gangen wird ${ }^{22}$. So darf man den kulinarischen Schlußakkord des Prométhée mal enchainné, die Verspeisung des Adlers, getrost als groteske Inszenierung des Abendmahls, d. h. als Christus-Parodie verstehen. In dieser Beziehung bewegt sich Gide durchaus auf der von Michail Bachtin skizzierten karnevalistischen Linie des Dialogischen. Das Heilige geht mit dem Unheiligen, das autoritäre Wort mit dem subversiven eine ebenso widersprüchliche wie ästhetisch produktive Allianz ein ${ }^{23}$. In der Doppelbödigkeit und Widersprüchlichkeit der Vertextungsstrategien äußert sich zwar nicht unbedingt - wie bei Bachtin der soziale, aber doch der ethische Impetus seiner Poetik, denn Dialogismus, Stimmenvielfalt, antizentralistische und permanent subversive Rede sind mit der freiheitlichen Lebensauffassung Gides aufs engste verbunden ${ }^{24}$.

Wenn gerade von Doppelbödigkeit und Dialog die Rede war, so kann man das ganz konkret auf die genannten Wirkungsformen beziehen. Remin is zenzen erscheinen bei Gide vor allem als inhaltliche bzw. stoffliche Anspiclung auf die mythische Vorgeschichte seines Prometheus. Wenn dieser als fabricant d'allumettes sans brevet, als Streichholzfabrikant, der keine Lizenz besitzt, ins Gefängnis muß, steht im Hintergrund zunächst kein spezifischer Text, kein bestimmter Autor, sondern gleichsam die Tradition aller Texte, die dem mythischen Stoff verpflichtet sind. Dergleichen Reminiszenzen spielen permanent in das vom Erzähler ausdrücklich auf die aktuelle Gegenwar bezogene Geschehen hinein: Gides Erzählung beginnt wie ein historischer Bericht (9): Au mois de mai 189..., deux beures après midi, on vit ceci qui put paraître étrange. Was dann kommt, hat freilich mit einer "Chronik" nicht mehr viel zu tun: Chronistische und anachronistische Schreibweise, Olymp und moderne Großstadt, mythischer Kaukasus und Pariser Café-Atmosphäre, Zeus als Kapitalist oder Prometheus als Flaneur: der Mythos durchkreuzt im wahrsten Sinne des Wortes das Bemühen des Erzählers, die Authentizität der geschilderten ,faits divers ${ }^{c}$ formal (als chronikalische Darstellung) abzusichern. Die historische Spanne, die den Gide-Text von der antiken, klassizistischen oder romantischen 'Tradition trennt, ist mit ausschlaggebend für den Grad der Transformation: Äußerlich ablesbar an der neuen raumzeitlichen Umgebung und den handlungsmäßigen Veränderungen, wie sie insbesondere im Verhältnis zwischen Prometheus und „seinem“ Adler - das Possessivum hat hier eine hermeneutische Schlüsselfunktion - zutage treten.

Man sollte solche modernen Transformationen des Mythos aber nicht undialektisch auffassen: Gegen den in diesem Zusammenhang benutzten Ter-

${ }^{22}$ Vgl. ausführlicher zur mittelalterlichen Tradition der Sottie: Barbara Goth: Unters. zur Gattungsgesch. der Sottie, München 1967.

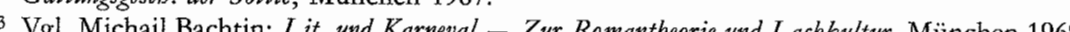
Bal Minch Bachtins Dialog-Begriff und die Sottie stehen auch im Mittelpunkt der Untersuchun von Jean Claude Aubailly: Le Monologue, le Dialogue et la Sotie, Paris 1976.

Eine systematische Untersuchung karnevalistischer Elemente im Werke Gides steht noch aus. Erste Ansätze zeigen, daß eine solche Untersuchung - insbesondere im Zusammenhang mit dem Phänomen der Intertextualität und der Wiedereinführung der Sottie bei Gide - lohnend wäre. Vgl. dazu Daniel Moutote: Intertextualité et journal dans l'cump d'André Gide, in: Le plaisir de l'intertexte, aaO. [Anm. 9] 137-184. 
minus „Schwundstufe“ spricht das Faktum kontinuierlicher historischer Anwendbarkeit ${ }^{25}$. Daß beispielsweise Prometheus, der Feuerbringer, während seines Vortrags ein Feuerwerk veranstaltet, zur pyrotechnischen Ablenkung des Publikums, wie er sagt, ist einer von vielen Belegen dafür, wie geschichtlich konsequentes Weiterdenken und Demontage des Prometheus-Mythos in diesem Text auf paradoxe Weise ineinandergreifen. Die durch solche intertextuellen Praktiken erzeugten komischen Effekte - die neben der Ambivalenz und der Unabgeschlossenheit die Qualität des dialogischen Wortes ausmachen werden freilich als solche erst nachvollziehbar, wenn der Leser das Heroische und den Pathos früherer Prometheus-Bearbeitungen gleichsam mitdenkt.

Darüber hinaus bedient sich Gide des Zitats, also der wörtlichen Wiederholung, um seinen ,neuen' Prometheus von der prometheischen Tradition abzuheben. Er greift jedoch in diesem Fall weniger auf Texte zurück, durch die der Prometheus-Stoff überliefert wurde - man findet beispielsweise nur Goethe- oder Shelley-Reminiszenzen, aber keine direkten Zitate -, sondern läßt seinen Prometheus die Bibel zitieren, u. a. während der Begräbnisrede für Damoclès (103): Messieurs, qui voulez bien m'écouter, les paroles de l'Ecriture qui serviront de texte à mon bref discours d'aujourd'bui sont celles ci: Laissez les morts ensevelir les morts. Dem Matthäus-Evangelium (8:22) entnommen und in einen neuen Kontext transferiert, bekommt das Zitat eine neue Sinnfunktion, so wie umgekehrt der aufnehmende Text durch die rezipierte Erscheinung fremdbestimmt wird. Diese Dialektik läßt sich mit Antoine Compagnon auf folgende Formel bringen ${ }^{26}$ : „La citation travaille le texte, le texte travaille la citation“. Es wird gleich noch darüber zu sprechen sein, welche Rolle die Bibel in der Gideschen Prometheus-Fassung hermeneutisch spielt; für jetzt sei festgestellt, $\mathrm{da} B$ sie in der Auslegung des Mythos eine vermittelnde und kommentierende Funktion übernimmt. Der Mythos wiederum leistet Hilfestellung bei Gides Auseinandersetzung mit dem Christentum.

Als drittes wichtiges Element intertextueller Produktion muß die Pa rodie genannt werden. In der als Geschichte in der Geschichte präsentierten parabolischen Histoire de Tityre ist sie unübersehbar, da sie sich auf Texte bezieht, die auch der nicht so eifrige Bibelleser im Ohr haben dürfte: Das Gleichnis, das im übrigen schon als Gattung intertextuell mit der Bibel verflochten ist (Systemreferenz), parodiert u. a. das Johannes-Evangelium (104): Au commencement était Tityre. Et Tityre étant seul s'ennuyait, complètement entouré de marais. Or, Ménalque vint à passer, qui mit une idée dans le cerveau de Tityre, une graine dans le marais devant lui. Et cette idée était la graine, et cette graine était l'Idée. Diese Sequenz sucht sich ihren Zusammenhang einerseits in der horizontalen Ver-

${ }^{25}$ Karlheinz Stierle: Mythos als, bricolage $e^{4}$ and apei Endstufen des Prometbeusmythos, in: Terror und Spiel, hg. v. M. Fuhrmann (= Poetik und Hermeneutik IV), München 1971, 455-472. Einen ähnlichen Vorbehalt kann man in bezug auf die Formulierung von Hans Blumenberg anmelden: „Prometheus, der Menschentöpfer, ist auch an diesem Ende seines Mythos, das Gide ihm zugedacht hat, nochmals an der Menschwerdung des Menschen beteiligt" H. B.: Arbeit am Mytbos, Frankfurt(M. 1979, 682

${ }^{26}$ La seconde main - ou le travail de la citation, Paris $1979,37$. laufsrichtung des Textes, indem sie die Lektion des Prometheus erhellt. Was fü Prometheus der Adler, das ist für 'Tityre das Samenkorn. Aus dem Samenkorn entsteht eine wuchernde Eiche. Immer kompliziertere Arbeitsvorgänge, ja ein ganzer Verwaltungsapparat werden notwendig, um das Wachstum der Eiche unter Kontrolle zu halten. Bis Tityre beschließt, die Eiche, die sein Leben aufzuzehren droht, zu ignorieren und wieder in seinen Sumpf zurückzukehren. Gleichzeitig funktioniert die Histoire de Tityre in vertikaler Verlaufsrichtung, indem sie den Leser gewissermaßen zu Ausflügen in die Kulturgeschichte einlädt: Prometheus-Überlieferungen, Bibel, Vergils Eklogen, die übrigens auch in vielen anderen Texten Gides eine Rolle spielen, u. a. m. ${ }^{27}$.

So kompliziert und uneinheitlich die Vertextungsstrategien sind - die Titelparodie (mal enchaîné) verweist zugleich symbolisch auf die Art der künstlerischen Gestaltung - so vielschichtig und in mancher Beziehung widersprüchlich scheint das Prometheus-Bild, das der Text als Ganzes vermittelt. Die Polyvalenz ist teilweise in der Symbol-Qualität des Mythos schon vorgegeben, sie läßt sich mit Raymond Trousson auf die Formel bringen ${ }^{28}$ : qui dit Prométbée, pense liberté, génie, progrès, connaissance, révolte. Alle diese symbolischen Bestimmungen spielen in der Gideschen Prometheus-Fassung aber als immer schon vermittelte, das heißt in ihrer Dependenz von bestimmten geschichtlich geprägten Auffassungsakten eine Rolle. Gides Prometheus-Bearbeitung hat also zunächst ihren Stellenwert als ironische Auseinandersetzung mit vorgegebenen Deutungsmustern. So ruft er beispielsweise mit der Shelley-Reminiszenz noch einmal die arkadische Urwüchsigkeit, den Zustand vor der Vertreibung aus dem Paradies in Erinnerung (67-68): Sur les flancs ruisselants du Caucase, beureuse et nue aussi la lascive Asia m'embrassait. Ensemble nous roulions dans les vallées; nous sentions l'air chanter, l'eau rire, les plus simples fleurs embaumer. [... Soudain, un jour, Asia me dit: tu devrais t'occuper des bommes. Il me fallut d'abord les cbercher. Je voulus bien m'occuper d'eux; mais c'était en avoir pitié. Ils étaient très peu éclairés; j'inventai pour eux quelques feux; et dès lors commença mon aigle. C'est depuis ce jour que je m'apergois que je suis nu.

Die unterlegten Intertexte dieses Abschnitts stehen ihrerseits in FrageAntwort-Relation zueinander, kommentieren sich gegenseitig. Von den GenesisMotiven der Suche (Adam, wo bist du?) und der Nacktheit (und sie wurden gewabr, daß sie nackt waren) zum christianisierten Prometheus Shelleys (der mitleidende Prometheus, dic Auseinandersetzung mit dem Bösen). Vom Fortschrittspessimismus à la Rousseau zur romantischen Konzeption prometheischer Revolte. Prometheus-Faszination versus Prometheus-Schelte. Fortschritt und Bewußt-

27 Dazu eine vielleicht etwas spitzfindige Beobachtung: Am Anfang von Vergils Bucolica steht: Tityrus, du rubst bier unterm Dach breitastiger Bucbe [...] Wenn Gide schreibt (104), Au commencement était Tityre, so könnte es sich dabei also um eine doppelte Anspielung (auf die Bibel und auf den Anfangsvers der Bucolica) handeln. Im übrigen produziert da Gide-Gleichnis ständig wechselseitige Kontaminationen von Bibel- und Vergil-Text. Der intertextuelle Zusammenhang ergibt sich darüber hinaus aus der gemeinsamen Thematik, z. B: ,Natur' vs. ,Kultur', ,Idylle' vs. ,Zwietracht' etc.

${ }^{28}$ Raymond Trousson: Le thème de Prométbée dans la litt. europ., Genf 1964, Tome I, IV.

arcadia 23 
sein als die Wurzel allen Übels (70-71): La croyance au progrès, Messieurs, c'était leur aigle.

In diese deutlich ideologisch aufgeladene Diskussion müssen wir auch Gides unmittelbaren literarischen und außerliterarischen Kontext einbeziehen. Pathetische Verteidigung der Wissenschaft, aber auch religiöser Messianismus gehören mit zu den typischen Merkmalen eines im letzten Drittel des 19. Jahr hunderts überall in Europa zu beobachtenden Prometheus-Booms. Titel wie Le Mystère du Progrès (Saint Yves, 1878) oder Le Prométhée de l'avenir (Delarue $1895)^{29}$ sprechen für sich. Vor dem Hintergrund solcher neo-klassizistischer 5-Akt-Tragödien wird der kontestative Charakter der Gideschen Bearbeitung besonders deutlich. (Vgl. auch die Umwertung der traditionellen Licht-Symbolik: Streichhölzer und Leuchtraketen als im wahrsten Sinne des Wortes erhel lende Symbole.)

Wie anfangs schon angedeutet wurde, zielt aber Gides Prométhée mal enchaîne über die innerliterarische Auseinandersetzung hinaus. Für die Art und Weise, wie er den Prometheus-Stoff gestaltet, ist unter anderem der familiäre, und das bedeutet in diesem Fall der religiöse Hintergrund entscheidend. Die streng protestantische Erziehung, die der junge Gide nach dem frühen Tode seines Vaters durch die Mutter erfahren hat, prägte ihn so, daß er als zwanzig jähriger die Salons der Symbolisten angeblich nur mit der Bibel in der Tasche betrat ${ }^{30}$. Um so radikaler erscheint der Bruch mit der religiösen Vergangenheit nach 1895. Ein Prometheus ohne Ketten und Adler, das ist die Wendung gegen die formules restrictives der Kirche ${ }^{31}$, gegen die puritanische Moral, gegen alle institutionalisierten Formen des Christentums, das ist die Entscheidung für einen individuellen, aktiven Humanismus, der keiner göttlichen Unterstützung bedarf.

Andererseits gewinnt man den Eindruck, daß sich Gides Bekenntnis zur moralité privée und zum absoluten Individualismus mit bestimmten Vorstellunon chereinbaren läßt, ja auf diesen beruht, nämlich dort, wo sein Prometheus allegorisch auf politische Zusammenhänge anspielt. Gide besaß einen ausgeprägten Sinn für politische Moral, und wo diese, wic in der Dreyfus-Affaire, in Frage gestellt war, da meldete er sich auf seine Weise zu Wort ${ }^{32}$. Es gibt Stellen im Text, wo der politische den religiösen oder metaphysischen Code gleichsam zu verdrängen scheint (84): Mon action sur Paris est cacbée [...], bestätigt Zeus, je lance. Puis, une fois une affaire lancée, je la laisse. In entsprechende Machenschaften, freilich als Opfer, ist der im Gefängnis einsitzende Prometheus verwickelt (50): De quoi m'accuse-t-on?, fragt er. Gide, erklärter ,Dreyfusard` wie Zola und die meisten Intellektuellen, hat

${ }^{29}$ Vgl. ebd. II $413-417$.

30 Vgl. Germaine Brée: André Gide, Paris 1953, 21.

31 Vgl. Gide: Journal 1889-1939, aaO. [Anm. 14] 95: Mais catholicisme d'abord, protestantisme ensuite, après avoir été formules expansives, sont formules restrictives depuis longtemps; gaines dures et coquilles où l"esprit se gêne.

32 Zur Evokation der Dreyfus-Affaire vgl. Pierre Masson: ,Le Prométhée mal enchainét ou Du détournement d'un mytbe à des fins personelles, in: Bull. des amis d'André Gide, aaO. [Anm. 21] $5-29$, hier $6 \mathrm{ff}$. in seinen Text Schlüsselbegriffe eingestreut, die im Jahre 1899, also während die öffentliche Diskussion um den jüdischen Hauptmann Dreyfus und seinen angeblichen Verrat ihren Höhepunkt erreicht, von einiger Brisanz sein mußten: Prison, scandale, affaire etc. Wenn derartige Konstruktionen darauf schließen lassen, daß zeitgeschichtliche Bezüge unmittelbar in die produktive Auseinan dersetzung mit dem Prometheus-Mythos hineingewirkt haben, so läßt sich Gide andererseits mit dem Zola des J'accuse ...! kaum auf eine Stufe stellen. Sein Engagement bleibt zumindest in diesem Zusammenhang äußerst ambivalent, folgt keiner in sich kohärenten Argumentation, sondern erschöpft sich wie im Falle der Auseinandersetzung mit den literarischen Mustern - in der Anspielung. Die spielhaften und ironischen Momente bestimmen freilich die Gesamtstruktur dieser sotie, die in ihrer inneren Dialogizität recht genau das ausdrückt, was Gide selber einmal als die Pendelbewegung des Denkens (le balancement de ma pensée) bezeichnet hat ${ }^{33}$ : Elle se développerait en dialogue [...] et pousserait des branches à la fois dans des directions opposées. C'est seulement ainsi que je pourrais à peu près me satisfaire.

III

Eine kurze Zwischenbilanz: Die Analyse sollte unsere grundsätzlichen Erwägungen zum komparatistischen Stellenwert intertextueller Prozesse bestätigen. Drei Fragenkomplexe sind durch die Prometheus-Bearbeitung, und insofern ist sie wirklich exemplarisch, vorgegeben, nämlich das Problem der Identifizierung fremder literarischer Äußerung in einem gegebenen Text, die mit der Transposition verbundenen strukturellen Probleme sowie die Frage nach der Funktion. Daß man sich zunächst einmal Klarheit über die materielle Substanz der Intertextualität verschafft, scheint besonders dringlich. Nicht nur bei Gide bot es sich an, von der konkreten, empirisch nachweisbaren LektüreErfahrung, von den Kontakt-Beziehungen, wie Durišin sie bezeichnet, auszugehen. Wir halten es prinzipiell für gefährlich, mit einem abstrakten, maximalistischen Intertextualitätsbegriff zu operieren, der dem unkontrollierten Vergleich Tür und Tor öffnet. Das schließt nicht aus, daß man sich über die immense Reichweite intertextueller Verflechtungen theoretisch Gedanken macht. Die These, kein Text, der nicht zugleich Intertext wäre (Charles Grivel), ist zumindest nicht zu widerlegen. Davon versuchen uns - wie das kurze Zitat aus Max Frischs Stiller beweist - nicht zuletzt die Betroffenen selber zu überzeugen. Aber nicht mystische, sich irgendwo im Ungewissen verlierende Filiationen oder assoziative Bezugsherstellung zwischen Texten liefern die Basis für eine sinnvolle Auseinandersetzung mit dem Phänomen, sondern vielmehr das, was man heute gemeinhin als produktive Rezeption bezeichnet. Letztere schließt alle konkreten literarischen Wirkungsformen ein, d.h. außer der Parodie, die in Bachtins Konzept des Dialogischen eine etwas einseitige Rolle spielt, auch die ennsthaften Transpositionen, die Reminiszenz, das Zitat,

${ }^{33}$ Tagebucheintragung vom 12. Jan. 1941. Vgl. Journal 1939-1949 - Souvenirs, Paris (Bibl. de la Pléiade) 1954, 64-65. 
das Motto usw. Von Intertextualität sollte man also sprechen, wenn ein gegebener literarischer Text in seiner semantischen und/oder syntaktischen Struktur durch eine oder mehrere fremde literarische Äußerungen (oder durch ein bestimmtes textuelles System) nachweislich mitbedingt wird, wobei sich der ursprüngliche Wert dieser Äußerung bei der Eingliederung in die neue Struktur verändert ${ }^{34}$. Diese strukturelle Leistung konnten wir an der Gideschen Prometheus-Bearbeitung ebenso ablesen wie das, was man seit Gadamer als die Applikation ästhetischer Erfahrung, nämlich als die „Anwendung des zu verstehenden Textes auf die gegenwärtige Situation des Interpreten bezeichnet ${ }^{35}$. Der Dialog zwischen den Texten vollzieht sich nicht als zeitunabhängiger, freischwebender Prozeß, sondern ist, wie gezeigt wurde, nach Inhalt und Form historisch und individuell determiniert. Ein Prometheus im Paris der Jahrhundertwende ist zugleich Ausdruck der Widersprüche, die diese Epoche kennzeichnen. Das gilt einerseits für den ideellen Bereich, den im Text ausgetragenen Konflikt zwischen alter und neuer, öffentlicher und privater Moral, zwischen christlichem Glaubensdogma und anti-religiöser Einstellung, autoritaristischem und liberalistischem Denkansatz. In seiner ideologischen Komponente profitiert Gides nach totaler Autonomie strebender Prometheus sicherlich auch von dem durch Nietzsche mitgeprägten geistigen Klima der Zeit ${ }^{36}$.

Andererseits gilt es, die literarischen Entwicklungstendenzen des 19. Jahrhunderts in eine Funktionsanalyse intertextueller Praxis mit einzubeziehen. Von dem Prometheus-Boom, zu dem Gide einen Anti-Prometheus liefert, war schon die Rede. Hinzu kommt, daß er den im klassischen Sinne, gepflegten narrativen Diskurs der Realisten und Naturalisten auf spektakuläre Weise verabschiedet. Die intertextuelle Reflexion verselbständigt sich, Ambivalenz, Offenheit, ,work in progress` etc. bestimmen die neue erzählerische Praxis Auch deshalb darf man den komparatistischen Stellenwert dieses Prometheus nicht auf den binären Vergleich mit bestimmten kulturellen Gipfelleistungen, mit Dichter-Vätern wie Goethe, Shelley oder Dostoevskij reduzieren. Intertextualität und innere Dialogizität verstehen sich hier - und das läßt sich mi Blick auf die literarische Avantgarde des 20. Jahrhunderts bis hin zur zeitgenössischen Literatur verallgemeinern - als bewußt eingesetzte künstlerische Verfahren, als ein Versuch, die Kunst von normativen Beschränkungen zu befreien.

In diesem Sinne ist André Gide nun in der 'Tat ein moderner' Autor. Und wenn es stimmt, daß gesteigerte, $d . h$. bewußt praktizierte, sich vervielfachende

34 Vgl. D̆urišin: Vgl. Lit.forscbung, aaO. [Anm. 12] 142.

Wabrbeit und Metbode, Tübingen ${ }^{21965,} 291$

${ }^{36}$ Die Beziehung zwischen Gide und Nietzsche beschränkt sich natüllich nicht auf, Klimatisches'. Der Roman L'Immoraliste und viele andere Texte sind z. T. sehr direkt durch Gides Nietzsche-Erlcbnis geprägt. An Nietzsche erinnert in Le Prométbée mal encbaîné u. a. dic Idee der moralité privée. André Moutote deutet den ersten Teil der Prometheus-Ansprache u. a. als Zaratbustra-Parodie (Le journal d'André Gide et les problèmes du Moi, Paris 1968, 99 [Anm. 10]). H. Blumenberg behauptet, Zeus (der Bankier) sei „die Karikatur von Nietzsches Übermenschen", aaO. [Anm. 25].
Intertextualität ${ }^{37}$ ein Signum ,postmoderner ${ }^{6}$ Schreibweise darstellt, so nimm der Prometheus von 1899 vorweg, was offenbar historisch noch gar nicht sein darf. Aber ungeachtet der Diskussion um historische Begriffe scheint man sich in der Sache einig zu sein: daß nämlich das Erzählen, in diesem Jahrhundert zumal, immer mehr wie ein „entfesseltes Gespräch“ funktioniert. Die Formulierung - sie stammt von Rolf Kloepfer ${ }^{38}$ und geht auf Bachtins Theorie der Redevielfalt im Roman zurück - kennzeichnet ziemlich genau den Dialog-Charakter unseres Textbeispiels. Was „die Nutzung aller außerliterarischen und literarischen Textsorten und ihrer Inhalte ${ }^{\star 39}$ betrifft, so repräsentiert sich uns der , schlechtgefesselte prometheische Held (wie vormals unter ganz anderen Voraussetzungen die prometheischen Künstlerfiguren bei Shaftesbury oder Goethe) ja geradezu als ein Symbol für solche künstlerischen Entgrenzungsvorgänge. Gide hatte die Prometheus-Bearbeitung mit einer Widmung für Paul-Albert Laurens versehen; er vergleicht bei dieser Gelegenheit sein Werk mit wildem Hafer (cette gerbe de folle ivraie), mit Unkraut also. Der wie Unkraut wuchernde Text, der Autor als Entfesselungskünstler solche Metaphorik darf man getrost ironisch auffassen: Wir haben uns davon überzeugen können, daß sich hinter dem scheinbar Zufälligen, Ungeordneten und Widersprüchlichen dieser von Intertextualität wie durchtränkten Erzählung ein erhebliches $\mathrm{Maß}$ an Rationalität, an systematischem Textbewußtsein und künstlerischer Experimentierfreude verbirgt.
37 Vgl. dazu unter narratologischen Gesichtspunkten vom Vf.: Der labyrintbische Diskurs Vom Mythos zum Eräälmodell, Frankfurt/M. 1987, bes. $306 \mathrm{ff}$.

38 Rolf Kloepfer: Der Roman als entfesseltes Gespräcb, in: Erzä̈blung und Erzäblforscbung im 20. Jh., hg. v. R. Kloepfer und G. Janetzke-Dillner, Stuttgart/Berlin/Köln/Mainz 1981. 